\title{
ECOEFICIÊNCIA COMO ESTRATÉGIA DE GESTÃO PARA A SUSTENTABILIDADE EMPRESARIAL: UMA ANÁLISE DA EMPRESA NOOCITY
}

\author{
Anaísa Filmiano Andrade Lopes ${ }^{1}$
}

\author{
Diogo Sá da Silva Pompeu²
}

\begin{abstract}
RESUMO
Atualmente o ramo empresarial está implementando estratégias para melhoria da sua imagem institucional e da qualidade de seus serviços. Contudo, são necessárias novas formas de organização, como a sustentabilidade empresarial, proposição que inova e desafia organizações e administradores a respeito da necessidade de conciliar as dimensões econômica, ambiental e social nos negócios. Assim, este artigo objetiva evidenciar como a sustentabilidade empresarial, através da ecoeficiência, pode gerar benefícios para o ambiente, apontando como exemplo, as estratégias adotadas pela empresa Noocity. O procedimento metodológico foi pautado em análise bibliográfica, para tanto, foi explanado definições sobre o tema, caracterizando-se como um artigo de revisão. Os resultados apontam que a empresa Noocity desenvolve técnicas ecoeficientes para a construção de hortas orgânicas como a Noocity Growbed e o Growpocket. Conclui-se que a mesma visa interagir o desempenho econômico e o desempenho ambiental, de modo a criar e promover valores com menor impacto sobre o meio ambiente.
\end{abstract}

PALAVRAS-CHAVE: Sustentabilidade Empresarial. Ecoeficiência. Hortas Orgânicas. Noocity.

\section{ECO-EFFICIENCY AS MANAGEMENT STRATEGY FOR CORPORATE SUSTAINABILITY: AN ANALYSIS OF NOOCITY COMPANY}

\begin{abstract}
Currently the business branch is implementing strategies to improve their corporate image and quality of its services. However, they require new forms of organization such as corporate sustainability, proposition that innovates and challenges organizations and administrators about the need to reconcile the economic, environmental and social business. Thus, this article aims to show how corporate sustainability through eco-efficiency, can generate benefits for the environment, pointing as an example, the strategies adopted by Noocity company. The

\footnotetext{
${ }^{1}$ Mestranda em Arquitetura e Urbanismo, Universidade Federal de Uberlândia - UFU. ana_isaandrade@hotmail.com

${ }^{2}$ Mestrando em Geografia, Universidade Federal de Uberlândia - UFU. diogo_geoufu@yahoo.com.br
} 


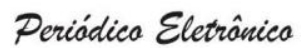

methodological procedure was guided by literature review, therefore, it was explained definitions of the subject, characterized as a review article. The results show that the Noocity company develops eco-efficient techniques for building organic gardens as Noocity Growbed and Growpocket. It concludes that it aims to interact economic performance and environmental performance in order to create and promote values with less impact on the environment.

KEYWORDS: Corporate Sustainability. Eco-efficiency. Organic gardens. Noocity.

\section{ECOEFICIENCIA COMO ESTRATEGIA DE GESTIÓN PARA LA SOSTENIBILIDAD EMPRESARIAL: UN ANÁLISIS DE LA EMPRESA NOOCITY}

\section{RESUMEN}

Actualmente la rama de negocios está implementando estrategias para mejorar su imagen corporativa y la calidad de sus servicios. Sin embargo, requieren nuevas formas de organización como la sostenibilidad corporativa, proposición que innova y desafía a las organizaciones y los administradores acerca de la necesidad de conciliar el negocio económico, ambiental y social. Por lo tanto, este artículo tiene como objetivo mostrar cómo la sostenibilidad corporativa a través de la eco-eficiencia, puede generar beneficios para el ambiente, señalando como ejemplo, las estrategias adoptadas por la compañía Noocity. El procedimiento metodológico fue guiado por revisión de la literatura, las definiciones, por lo tanto, se explicó de la asignatura, que se caracteriza como un artículo de revisión. Los resultados muestran que la compañía Noocity desarrolla técnicas ecoeficientes para la construcción de jardines orgánicos como Noocity Growbed y Growpocket. La conclusión es que su objetivo es interactuar desempeño económico y el desempeño ambiental con el fin de crear y promover los valores con un menor impacto sobre el medio ambiente.

PALAVRAS-CLAVE: Sostenibilidad Corporativa. Eco-eficiencia. Jardines orgánicos. Noocity.

\section{INTRODUÇÃO}

A redemocratização do Brasil a partir da década de 1980 estabeleceu critérios, ainda que superficiais, de sustentabilidade. Uma série de leis ambientais criadas nesse período permite reconhecer a necessidade de um processo de sustentabilidade e desenvolvimento sustentável no país (ALEXANDRE; KRISCHKE, 2006). A Política Nacional do Meio Ambiente (1981) tornou obrigatória a avaliação de impacto ambiental e o licenciamento das atividades poluidoras; a Constituição Federal (1988) incorporou no artigo 225 questões relacionadas ao Meio Ambiente, impondo ao Poder Público e à coletividade o dever de defendê-lo, possibilitando mais tarde a criação de leis específicas. 


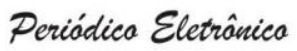

Em razão desse marco regulatório, não é raro observar nos documentos publicados pelas empresas (relatórios, propagandas e endereços eletrônicos) a declaração da implementação de estratégias de desenvolvimento sustentável. Ainda que o fenômeno da sustentabilidade possa ser considerado recente e desigual, quando se compara sua implantação nos diferentes setores econômicos, é incontestável a expansão da introdução de modelos de gestão ambiental nas empresas brasileiras como forma de consolidação de suas políticas de desenvolvimento sustentável.

Conforme Zamboni \& Ricco (2009), a efetividade e a evolução das teorias e práticas de desenvolvimento sustentável incluem, necessariamente, a participação de todos os atores sociais, dentre eles, as empresas. Assim, torna-se cada vez mais frequente e emergente a necessidade da reflexão do empresariado sobre o seu papel no desenvolvimento de nossa sociedade. Diante da conjuntura vivida nos últimos séculos, o desempenho do empresariado no contexto da revolução industrial, científica e tecnológica passou a ser, então, objeto de questionamentos, na medida em que a obsessão única e exclusiva pelo lucro foi e tem sido uma das mais lembradas características desse setor, o que, tem interferido negativamente em sua reputação, suscitando nas empresas novas maneiras de pensar e agir. Almeida (2002, p.53), nos apresenta esta situação da seguinte forma:

[...] Acostumado a dividir o universo em compartimentos estanques para poder entendê-lo - fruto de uma visão cartesiana, mecanicista, reducionista, forjada em trezentos anos de Revolução Científica e Industrial -, nos últimos anos do século XX o homem viu-se às voltas com a constatação de que a natureza não se deixa apreender completamente pelas ferramentas tradicionais de análise [...]. Para ser compreendida, pede um novo: orgânico, holístico, integrador [...].

Portanto, é cada vez mais necessária a busca por novas formas que contribuam não somente para os negócios, mas também para construção de uma sociedade sustentável. Investir em sustentabilidade empresarial é, além de um comportamento ético, uma maneira de, indiretamente, contribuir para a perenidade dos negócios, beneficiando no fim, a própria atividade empresarial (ZAMBONI \& RICCO, 2009). 
Diante desse contexto, a presente pesquisa objetiva apresentar as estratégias de sustentabilidade empresarial desenvolvidas pela empresa Noocity, a qual visa a implantação de hortas orgânicas em pequenos espaços urbanos. A relevância da pesquisa se justifica pela atualidade da temática, uma vez que, diante dos problemas ambientais, observa-se que as empresas têm procurado equacionar os objetivos da obtenção de lucros e da remuneração de seus acionistas adotando modelos de gestão que incluem práticas de sustentabilidade como forma de evidenciar suas potencialidades.

\section{METODOLOGIA}

Para o desenvolvimento metodológico foi realizado um levantamento bibliográfico das temáticas correlatadas à evolução da sustentabilidade, sustentabilidade empresarial, ecoeficiencia, hortas orgânicas e a empresa Noocity.

Foram realizadas leituras em livros, artigos, dissertações e em teses disponíveis eletronicamente. Foram utilizadas imagens (adquiridas em endereços eletrônicos) de hortas orgânicas desenvolvidas pela empresa estudada. As imagens são ilustrativas e abordam o tema em discussão com a finalidade de ampliar a interpretação e a construção de ideias.

Como primeira etapa abordou-se sobre a evolução histórica da sustentabilidade e consecutivamente sobre a ecoeficiência como ferramenta para a sustentabilidade empresarial. Em seguida, foi aplanado sobre os resultados e discussões e por fim as considerações.

Ressalta-se que a presente pesquisa se caracteriza como sendo bibliográfica, a mesma analisa a produção em determinada área temática, neste caso em específico sobre a sustentabilidade empresarial. Este tipo de pesquisa também propicia ao pesquisador tomar conhecimento, em uma única fonte, do que ocorreu ou está ocorrendo periodicamente no campo estudado, podendo substituir a consulta a uma série de outros trabalhos. As revisões bibliográficas podem também contribuir com sugestões de ideia para o 


\section{Periádica Eletrânica

desenvolvimento de novos projetos de pesquisa (NORONHA \& FERREIRA, 2000).

Quanto à sua natureza, se caracteriza como descritiva e exploratória devido à busca por esclarecimentos de como a sustentabilidade empresarial se manifesta e como é utilizada a estratégia ecoeficiente da empresa Noocity. A pesquisa apresenta ainda um referencial teórico apontando a evolução dos aspectos ligados à construção do conceito de sustentabilidade e desenvolvimento sustentável até a conexão com o tema de ecoeficiência empresarial.

Para Moreira (2004), esse tipo de estudo é uma ferramenta importante para a investigação, pois propicia ao pesquisador tomar conhecimento, do que ocorreu ou está ocorrendo periodicamente no campo estudado. Trata-se, portanto, de um tipo de texto que reúne e discute informações produzidas na área de estudo. Ou seja, é necessário tomar como base os avanços já realizados e, por que não, as limitações dos estudos anteriormente dedicados ao tema.

\section{REFERENCIAL TEÓRICO}

\subsection{EVOLUÇÃO HISTÓRICA DA SUSTENTABILIDADE}

A Organização das Nações Unidas - ONU - organizou, em 1972, em Estocolmo, Suécia, a I Conferência Internacional de Meio Ambiente e Desenvolvimento, onde oficializou a preocupação internacional com o meio ambiente e a necessidade de implementação de políticas públicas de meio ambiente (ANDRADE, 2005).

Em 1977, na cidade de Tbilisi, na Geórgia, ex-URSS, foi organizada a I Conferência Mundial de Educação Ambiental, vista como um manifesto em prol da restauração da Terra, como forma de garantir a sobrevivência de todas as espécies, e tendo o consumismo extremado, devido à industrialização capitalista, como um grande vilão nesse processo. No sentido de procurar uma nova ordem econômica mundial, na qual se tivesse um maior equilíbrio entre as 
dimensões econômica, social e ambiental, foi introduzido em 1987, no documento Nosso Futuro Comum (Relatório da Comissão Brundtland), o conceito de Desenvolvimento Sustentável, ou seja, um novo paradigma de desenvolvimento no qual as necessidades do presente são atendidas, sem comprometer a capacidade das futuras gerações de atenderem às suas próprias necessidades (ANDRADE, 2005).

Cerca de vinte anos após a Conferência de Estocolmo foi organizada pela ONU a II Conferência das Nações Unidas para o Meio Ambiente e o Desenvolvimento, ocorrida no Rio de Janeiro, Brasil, em 1992, conhecida como Rio-92 ou ECO-92, e que se tornou uma referência em relação à construção de acordos ambientais internacionais (ALMEIDA, 2002). O objetivo principal da Rio-92 foi buscar meios de conciliar o desenvolvimento socioeconômico com a conservação e proteção dos ecossistemas da Terra. Neste sentido, a RIO-92, deu origem à elaboração de vários documentos oficiais tais como: A Carta da Terra, aprovada posteriormente pela ONU em 2002; as Convenções Internacionais de Mudanças Climáticas, de Biodiversidade e de Desertificação; a Declaração de Princípios sobre Florestas e a Agenda 21, principal documento da Rio-92 e que serve de base para que cada país, estado, município e/ou instituição elabore seu plano de preservação para o meio ambiente.

A partir de então, várias outras conferências e acordos internacionais têm sido organizados de modo a promover o caráter de urgência na implementação do modelo de sustentabilidade em escala mundial. As empresas, os governos e a sociedade civil organizada, como principais atores desse processo, devem atuar conjuntamente de modo a encontrar o ponto de equilíbrio entre as dimensões econômica, social e ambiental em todas as suas iniciativas e atividades de modo a garantir o bem estar da sociedade e o futuro do próprio planeta.

\subsection{A ECOEFICIÊNCIA COMO FERRAMENTA PARA A SUSTENTABILIDADE EMPRESARIAL}


Empresas Sustentáveis são estabelecimentos públicos ou privados que tem como objetivo produzir bens materiais ou serviços com os conceitos de sustentabilidade. Primeiramente, para serem consideradas sustentáveis as empresas precisam modificar suas práticas diárias, aderindo ferramentas sustentáveis, como: reciclagem, tecnologias limpas, logística verde, energia limpa, hortas orgânicas, entre outras. A empresa deve ter preferência por fornecedores que também utilize dessas ferramentas (RODRIGUES, 2012).

Além disso, deve reforçar seu papel de agente estruturante na construção e desenvolvimento de uma comunidade equilibrada economicamente e socialmente. Entretanto, a sustentabilidade não pode ser encarada como uma meta a ser atingida dentro de um contexto finito, e sim, como um processo de evolução contínuo.

$\mathrm{Na}$ construção de sua sustentabilidade, a empresa deve estabelecer uma visão de planejamento e de operação, em todas as suas ações, que contemple o curto, o médio e o longo prazo (VAN BELLEN, 2007). Para tal, são fatores vitais para a empresa, a adoção de ferramentas como a ecoeficiência, que garanta à empresa uma atuação socialmente e ambientalmente responsável.

A estratégia voltada para a gestão da sustentabilidade da empresa combinando o desempenho econômico e o desempenho ambiental, de modo a criar e promover valores com menor impacto sobre o meio ambiente dá-se o nome de Ecoeficiência.

\begin{abstract}
A ecoeficiência atingi-se através da oferta de bens e serviços, que, por um lado, satisfaçam as necessidades humanas e contribuam para a qualidade de vida $e$, por outro, reduzam progressivamente 0 impacto ecológico e a intensidade de utilização de recursos ao longo do ciclo de vida, até atingirem um nível, que, pelo menos, respeite a capacidade de sustentação estimada para o planeta Terra (WBCSD apud, DIAS, 2006, p.130).
\end{abstract}

Este conceito foi introduzido como modelo de gerenciamento ambiental em 1992, pelo World Business Council for Sustainable Development (WBCSD). Atualmente, a Organization for Economic Co-Operation and Development (OCDE) e o WBCSD são os promotores mais atuantes dessa 
proposta de gestão ambiental. A implementação de práticas ecoeficientes é um processo de melhoria contínua, e para tal deve-se sempre ter em mente os limites e capacidades de um sistema resistir aos diferentes tipos de impactos ambientais. Almeida (2002, p.82) nos coloca que:

\begin{abstract}
Cabe às empresas, de qualquer porte, mobilizar sua capacidade de empreender e de criar para descobrir novas formas de produzir bens e serviços que gerem mais qualidade de vida para mais gente, com menos quantidade de recursos naturais. Ser ecoeficiente é oferecer bens e serviços que satisfaçam as necessidades, gerando impactos ecológicos menores e capazes de serem absorvidos pela natureza.
\end{abstract}

São muitas as oportunidades de as empresas serem ecoeficientes. Reorientação dos processos, revalorização dos subprodutos e a recolocação nos mercados podem apresentar um novo posicionamento estratégico, uma nova orientação mercadológica e também uma oportunidade para novos negócios (WBCSD, apud DIAS, 2000).

Essa nova postura - voltada para a conservação dos ecossistemas requer empenho, inovação e, acima de tudo, mudança. É neste sentido que as empresas, independentemente de porte e ramo de atuação, precisam ousar e sair de sua zona de conforto, rever seus modelos de produção, buscando outras maneiras de constituir e fazer negócios. A ecoeficiência se alcança pela entrega de produtos e serviços com preços competitivos que satisfaçam as necessidades humanas e melhorem a qualidade de vida, ao mesmo tempo em que seus impactos ecológicos são reduzidos a um nível equivalente à capacidade de carga do planeta (ALMEIDA, 2002).

Além disso, ela exige que as empresas tracem estratégias de gestão ambiental preventiva, que integrem aspectos ambientais ao ciclo de vida de seus produtos e serviços, relacionando a excelência ambiental com a empresarial. 


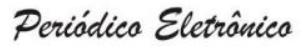

\section{RESULTADOS E DISCUSSÃO}

Diante da ampliação do debate em torno do tema Sustentabilidade, grandes empresas vêm se mobilizando na busca do alinhamento de suas práticas com valores ambientais.

A Noocity é uma empresa luso-brasileira dedicada a desenvolver soluções práticas e ecoeficientes para agricultura urbana. O nome Noocity surge da mistura entre o prefixo (noo) que representa a consciência coletiva e a palavra (city) que faz referencia o universo urbano. A empresa visa contribuir para uma sociedade cada vez mais consciente e autônoma, fornecendo as ferramentas necessárias para agir, reorganizando a paisagem urbana, a relação com a alimentação e a vida em comunidade (ARAÚJO, 2015).

\begin{abstract}
A ideia surgiu em 2013, quando os amigos José Ruivo, Pedro Monteiro e Samuel Rodrigues resolveram montar uma horta num pátio de um prédio no centro da cidade do Porto, Portugal. Por não encontrarem produtos adequados para agricultura urbana decidiram cultivar os seus próprios alimentos, juntaram esforços e experiências em arquitectura e permacultura e resolveram construir os seus equipamentos. No mesmo ano já partilhavam legumes e ervas aromáticas que cresciam aos montes numa série de caixas e sistemas onde antes só havia cimento. Os protótipos foram evoluindo e os três perceberam que poderiam transformá-los em produtos, a ideia amadureceu, tomou forma e ainda em 2013 nasceu oficialmente a Noocity Ecologia Urbana (NOOCITY, 2014, p.2).
\end{abstract}

A Noocity quer oferecer aos cidadãos urbanos a alternativa de criarem os seus próprios sistemas sustentáveis, mais parecidos com os processos cíclicos naturais, através de equipamentos eficientes e acessíveis que permitem às pessoas produzir alimentos na cidade de forma simples e ecológica, uma vez que segundo Cortez e Ortigoza (2009), o sistema de produção e consumo de alimentos atual é insustentável por ser um processo linear que, por um lado, consome e esgota recursos e, por outro, produz e acumula resíduos. Numa conjuntura de degradação ambiental e de cultura dos fast-foods, cuidar de nós mesmos e do meio ambiente permite caminhar em direção a cidades e comunidades mais saudáveis e sustentáveis (ARAÚJO, 2015). 
A estratégia da empresa busca repensar os espaços inutilizados e não produtivos (coberturas, varandas e pátios) e transformá-los em lugares de cultivo de alimentos, ou seja, busca eliminar as barreiras da agricultura urbana como a falta de espaço, falta de orientação e falta de tempo. Os produtos são leves e fáceis de transportar que permitem a instalação de uma horta em qualquer lugar, sem necessidade de obras ou utilização de ferramentas, adaptam-se a famílias, escolas e instituições. Os modelos são Growbed e Growpocket, que serão descritas nos próximos itens.

\subsection{GROWBED}

A Noocity Growbed é uma cama de cultivo (figura 1 e 2) com sistema de auto-rega (sub-irrigação), fácil de montar e de baixa manutenção, permitindo plantar uma grande variedade de legumes, frutos e ervas em qualquer lugar, é acessível a crianças e adultos, é portátil e não exige obras e ainda pode ser usada no interior dos imóveis (NOOCITY, 2014).

Figura 1: Sistema Growbed de Cultivo.

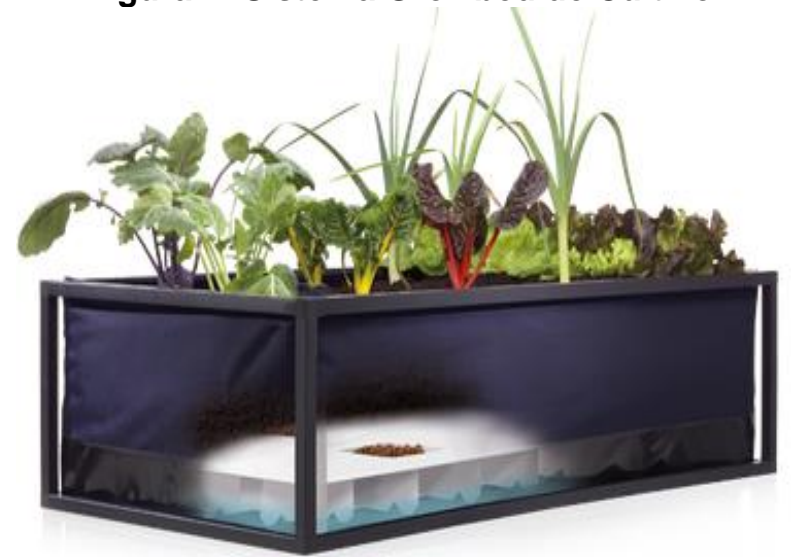

Fonte: http://www.noocity.com/produtos/growbed/
Figura 2: Growbeds em uma Cobertura.

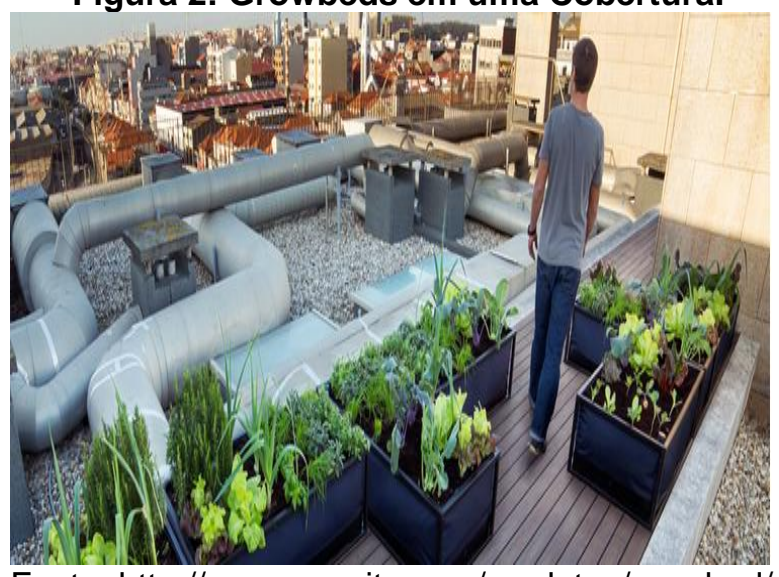

Fonte: http://www.noocity.com/produtos/growbed/

Este método apresenta o sistema de sub-irrigação com reservatório de água que diminui consideravelmente a evaporação e a frequencia da rega ${ }^{3}$.

\footnotetext{
${ }^{3}$ Para detalhes específicos, técnicos e de montagem da Growbed consultar o site da empresa: http://www.noocity.com/files/5414/1276/6923/Noocity_ManualGrowbed_digital_PT.pdf
} 
Considerado mais eficaz para o cultivo de alimentos em solo e especialmente indicado para agricultura urbana, o sistema de subirrigação consiste em fornecer água às plantas por baixo da zona de raízes. Através da ação da capilaridade, a água passa lentamente do reservatório para a zona de cultivo, conforme a necessidade das plantas. Uma caixa de ar separa a água do solo, permitindo às raízes o fácil acesso a oxigênio. $O$ resultado dessa configuração são plantas produtivas, economia de água e menos manutenção (NOOCITY, 2014, p.5).

Esta inovação previne problemas comuns como excesso ou falta de rega e reduz drasticamente a perda de água por evaporação ou drenagem, consumindo até $80 \%$ menos água do que os sistemas de cultivo convencionais (ARAÚJO, 2015).

\subsection{GROWPOCKET}

O Noocity Growpocket é um sistema de horta vertical ou horizontal (figura 3 e 4), com retenção de água, fácil de instalar e extremamente versátil, pois possui um duplo sistema de fixação que permite a utilização em qualquer lugar (paredes, varandas ou na própria Noocity Growbed - figura 5).

Figura 3: Growpocket Vertical.

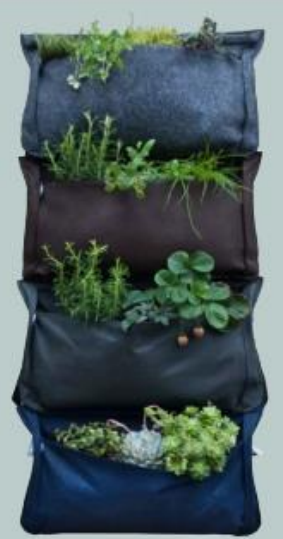

Fonte: http://www.noocity.com/files/PressKit.pdf
Figura 4: Growpocket Horizontal.

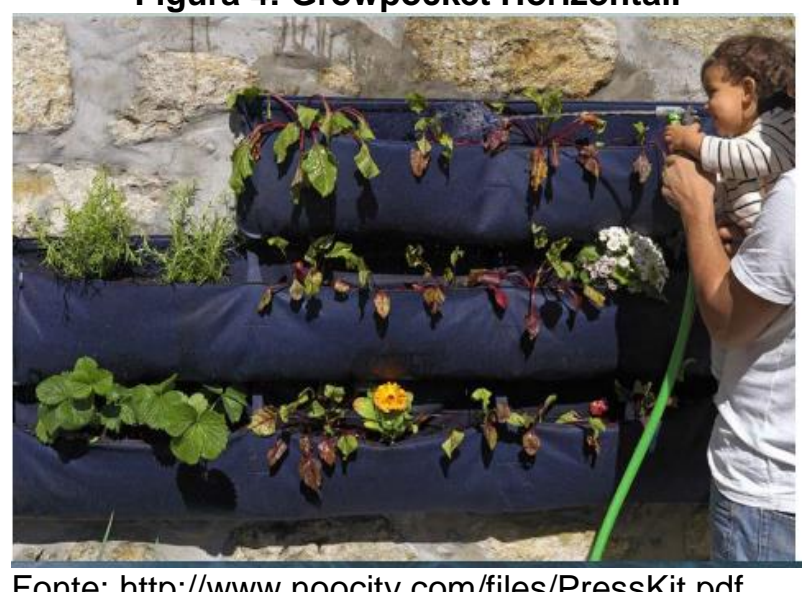

Fonte: http://www.noocity.com/files/PressKit.pdf 
Figura 5: Growpocket Fixada na Growbed.

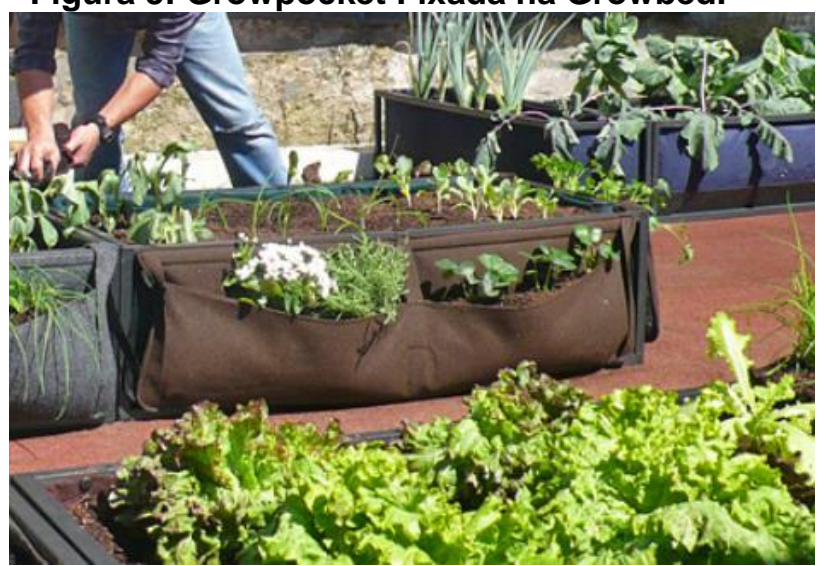

Fonte: http://www.noocity.com/files/PressKit.pdf

Desenhado para garantir a oxigenação do solo e o escoamento do excesso de água, possui uma tela impermeável que impede a passagem de água para a parede, o mesmo é dimensionado para o cultivo de plantas de pequeno e médio porte e pode ser mudado de local com facilidade ${ }^{4}$ (NOOCITY, 2014).

Assim, o growbed e growpocket são técnicas que promovem a produção agrícola orgânica, considerada ecológica e sustentável, pois respeita o meio ambiente, a biodiversidade e adota uma série de medidas preventivas para evitar a ocorrência de situações que obriguem ao uso de produtos químicos (fertilizantes, pesticidas e outros). As suas práticas prezam pela aproximação ao equilíbrio natural, pela maior utilização de fatores de produção renováveis e de baixo custo energético, e pela interdição de práticas e produtos de maior impacto ambiental. A agricultura orgânica integra um conjunto de técnicas agrícolas, visando a utilização racional do sistema formado pelo clima-águasolo-microorganismos-planta, de modo a preservar o equilíbrio dos ecossistemas agrícolas e torná-los sustentáveis a longo prazo (FUNDAÇÃO BIOLÓGICA, 2013).

A agricultura comercial utiliza produtos químicos e dispõe de técnicas voltadas para o cultivo dos vegetais em curto prazo. A ineficiência nutricional

\footnotetext{
${ }^{4}$ Para detalhes específicos, técnicos e de montagem da Growpockt consultar o site da empresa: http://www.noocity.com/files/5414/1276/6923/Noocity_ManualGrowbed_digital_PT.pdf
} 
dos vegetais cultivados nesse sistema é um dos fatores que influenciam na elaboração de uma horta orgânica, outros fatores importantes levados em consideração são o respeito com o meio ambiente e a aquisição de qualidade de vida.

Como verifica-se uma tendência cada vez maior dos seres humanos direcionarem-se as cidades, é fundamental discutir sobre as estratégias que aproveitem espaços urbanos considerados ociosos. A partir dos avanços tecnológicos e de um conjunto de idéias inovadoras, que na maioria das vezes, não necessitam de altos investimentos, como é o caso das hortas orgânicas, pode-se disseminar os ideais que privilegiam a sustentabilidade.

\section{CONSIDERAÇÕES FINAIS}

Para se efetivarem, as políticas e práticas de sustentabilidade empresarial devem, necessariamente, atender simultaneamente aos critérios de ecoeficiência e responsabilidade social. É neste sentido que se torna cada vez mais necessária a reflexão, disseminação e proposição de ideias e práticas que tornem realidade a sustentabilidade empresarial.

Em concordância com Leal et al. (2007), a construção de um futuro sustentável nas organizações passa rigorosamente pelo estabelecimento de mudanças corporativas e pela adoção de práticas gerenciais transparentes que valorizem as dimensões sociais e ambientais, melhorando a qualidade de vida, o bem-estar social, o equilíbrio econômico entre as nações e o respeito ao meio ambiente.

Diante do contexto, a sustentabilidade empresarial também deve ser vista como uma oportunidade de novos negócios para as empresas, esse foi o caso da empresa Noocity que vem se destacando ao conciliar progresso econômico, equidade social e preservação ambiental. Esta empresa está desenvolvendo ferramentas eficientes e acessíveis que permitem que as pessoas produzam hortas orgânicas nas cidades de forma simples e ecológica, colaborando para uma reorganização da paisagem urbana. $E$ através da plataforma online a mesma incentiva a troca de experiências, além de oferecer 


\section{Periódica Eletrânica

orientações práticas para apoiar aqueles que procuram um futuro sustentável para si e para o ambiente.

Pode-se concluir que a busca pela sustentabilidade empresarial pode gerar imagem e reputação, contribuindo também para o crescimento e perenidade dos negócios. Assim, é preciso repensar o significado do papel do empresariado, buscando ir além da geração de riqueza material, de forma que as empresas também exerçam a sua sustentabilidade empresarial, cooperando efetivamente para o desenvolvimento sustentável da humanidade.

\section{REFERÊNCIAS}

ARAÚJO, M. Sustentabilidade é ação: Campanha Growbed - Hortas Noocity. São PauloSP, $2015.2 \quad$ Disponível em: http://sustentabilidadenaoepalavraeaccao.com.br/2015/02/campanha-growbed-hortasnoocity.html. Acesso em 09 de setembro de 2015.

ALEXANDRE, A. F; KRISCHKE, P. J. Aspectos da institucionalização das políticas de sustentabilidade no Brasil. Revista Internacional Interdisciplinar Interthesis, Florianópolis, v. 3, n. 2, jul.,/dez., 2006.

ALMEIDA, Fernando. O bom negócio da sustentabilidade. Rio de Janeiro: Nova Fronteira, 2002.

ANDRADE, E. P. et al. (org.). Organizações sustentáveis - Contribuições a excelência da gestão. Rio de Janeiro: UFF/LATEC - ABEPRO, 2005.

BRASIL. Leis, Decretos. Lei o 6.938 de 31 de agosto de 1981. Dispõe sobre a Política Nacional do Meio Ambiente. Disponível em http://www.planalto. gov.br/ccivil_03/Leis/L6938.htm. Acesso em: 20 de agosto de 2013.

BRASIL. Constituição (1988). Constituição da República Federativa do Brasil: promulgada em 5 de outubro de 1988. 42. ed., atual. e ampl. São Paulo: Saraiva, 2009. 410 p. (Saraiva de Legislação).

CORTEZ, A.T.C.; ORTIGOZA, S.A.G. Da produção ao consumo: impactos socioambientais no espaço urbano [online]. São Paulo: Editora UNESP; São Paulo: Cultura Acadêmica, 2009. $146 \mathrm{p}$.

DIAS, Reinaldo. Gestão ambiental: responsabilidade social e sustentabilidade. São Paulo: Atlas, 2006.

FUNDAÇÃO BIOLÓGICA. Manual de Criação e Manutenção de uma horta biológica. Coimbra - Portugal, 2013. Disponível em: http://www.actuaracd.org/uploads/5/6/8/7/5687387/manual.biohorta.coimbra.pdf. Acesso em 09 de setembro de 2015.

LEAL, C. E., Chiganer, L., Xavier, J. C. Transformando Instituições Financeiras em Organizações Sustentáveis. Proceedings da Conferência Internacional SHEWC 2007, Santos, Brasil, em mídia digital. 
MOREIRA, W. Revisão de Literatura e Desenvolvimento Científico: Conceitos e Estratégias para Confecção. São Paulo, 2004.

NOOCITY. Noocity Growbed. Portugual, 2014. Disponível em: http://www.noocity.com/pt/produtos/growbed/. Acesso em 08 de setembro de 2015.

Noocity Growebed está sendo disponível para o Brasil. Portugual, 2014. Disponível em: http://www.noocity.com/pt/. Acesso em 08 de setembro de 2015.

NORONHA, D. P.; FERREIRA, S. M. S. P. Revisões da Literatura. In: Campello, B.S., Cendón, B.v. e Kremer, J.M. Fontes de Informação para Pesquisadores e Profissionais. Belo Horizonte: Ed. UFMG, p. 191-198, 2000.

RODRIGUES, F.A.; PAULETO, B. A.; SILVA R.F.S.; EUGENIO A. A Empresa como Modelo de Sustentabilidade. Revista @Alumni, v.2, no Especial, São Paulo - SP, 2012. Disponível em: http://fgh.escoladenegocios.info/revistaalumni/artigos/edEspecialMaio2012/vol2_noespecial_arti go_24.pdf. Acesso em: 08 de setembro de 2015.

VAN BELLEN, Hans Michel. Indicadores de sustentabilidade. Rio de Janeiro: FGV, 2007.

ZAMBON, Bruno Pagoto; RICCO, Adriana Sartório. Sustentabilidade Empresarial: uma oportunidade para novos negócios. Entrevista em 09 nov. 2009. Disponível em:

<http://www.craes.org.br/arquivo/artigoTecnico/Artigos_Sustentabilidade_Empresaria_Uma_op ortunidade_para_novos_negciosl.pdf>. Acesso em: $10 \mathrm{dez} .2014$. 